\title{
Detection of Cracking in Gear Teeth Using Acoustic Emission
}

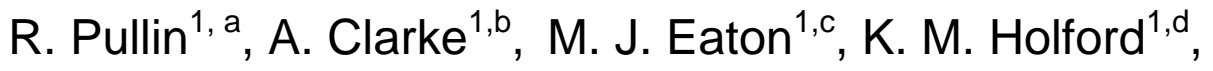 \\ S. L. Evans ${ }^{1, e}$ and J. P. McCory ${ }^{1, f}$ \\ ${ }^{1}$ Cardiff School of Engineering, Cardiff University, Queen's Buildings, \\ Cardiff, CF24 3AA, Wales, UK \\ apullinr@cf.ac.uk, bclarkea7@cf.ac.uk, eatonm@cf.ac.uk, dholford@cf.ac.uk, \\ evanss16@cardiff.ac.uk, ${ }^{\mathrm{f}} \mathrm{mcc}$ croryjp@cardiff.ac.uk
}

Keywords: Acoustic emission, gear teeth, structural health monitoring

\begin{abstract}
The detection of damage in gear teeth is paramount to any condition monitoring or structural health monitoring (SHM) tool for aerospace power transmissions such as those used in helicopters. Current inspection techniques include vibration analysis and time-inefficient visual inspection. Acoustic Emission (AE) is a very sensitive detection tool that has been successfully used in many SHM systems. Successful application of AE for damage detection in gear teeth will enable the optimisation of gear box design (and hence weight saving) in addition to safety improvements. This paper details a small aspect of a larger project designed to demonstrate automatic detection and location of common gear tooth defects. A novel test rig was designed to allow the fatigue loading of an individual gear tooth which was monitored using AE. The gear tooth was static in order to exclude the detection of AE signals arising from rotation; this allows initial development of the methodology prior to investigating rotating gears. Digital Image Correlation was used to determine the onset of cracking for comparison with the detected AE. Preliminary results of the investigation show that the developed methodology is appropriate for developing an automated gear health monitoring system and that future work should concentrate on the development of sensors and data acquisition methods associated with obtaining signals from rotating machinery.
\end{abstract}

\section{Introduction}

The development of detection and diagnostic methods for gear tooth faults in aerospace power transmission systems is an active research area. It is being driven largely by the interests of military organisations or large aerospace organisations such as NASA [1-3]. In certain situations, Health and Usage Monitoring (HUMS) systems are mandatory, such as helicopter operators servicing the North Sea oil and gas industry, and the majority of the commercial techniques are based on vibration methods $[4,5]$. In aerospace applications, the potential results of gear failure are serious, ranging from increased asset downtime and maintenance expenditure to, at worst, catastrophic failure with life-threatening consequences. Thus, new monitoring techniques which can identify early-stage failures are in demand.

Acoustic Emission (AE) monitoring is a technique which is used widely in other applications and which offers significant advantages in terms of early fault detection and diagnosis when compared with other techniques [6]. It is arguably the most sensitive NDT technique available, and relies upon the detection of stress waves generated by damage (cracks, plastic deformation etc) which propagate through the solid material as it undergoes strain. It is proposed that AE could offer significant advantages in gear monitoring applications. Recent advances at Cardiff University have led to novel techniques being developed to automatically to locate and identify damage in high noise environments $[7,8,9]$ in metallic structures. When compared with the reasonably mature application of AE to structural monitoring, the use of AE to monitor rotating machinery in general, and gears in particular, is still at the developmental stage, particularly when applications such as high speed, heavily loaded aerospace transmissions are considered. Workers have investigated the 
AE from spur gears $[10,11,12,13]$ but there remains much confusion surrounding the sources of acoustic emissions in gear contacts. The RMS approach used in the reported work has shown some modicum of success but further work is required not only to understand the source mechanisms and propagation but also to provide an automated technique for identifying damage in these types of applications.

In terms of commercial usage, AE monitoring of gear and bearing systems has been limited to relatively slow-speed application. For example, the Aquilla AE-Pro system [14] developed by the steelmaking company Corus is in commercial use for bearing monitoring at rotational speeds below $80 \mathrm{rpm}$.

The work presented here is part of a wider project which ultimately aims to develop a monitoring system for aerospace gears which provides a measurement of the health of the gear system, and is capable of monitoring lubricant health, asperity contact levels, surface degradation and root fillet cracking. Given the inherent complexities of measuring AE signals from a rotating gear pair, the current work involves a non-rotating pair of gears which are cyclically loaded in order to allow initial investigation into the AE signals produced by the initiation and propagation of a root fillet crack.

\section{Experimental Procedure}

A novel test rig was designed and manufactured to allow the fatigue loading of an individual gear tooth (Fig. 1). This allows a full AE analysis of damage signatures in fatigue crack growth in gear teeth without the presence of constant background noise due to rotational and frictional sources. This is evidently a simplified version of actual gear systems but allows techniques to be trialed and tested quickly whilst allowing validation using techniques such as digital image correlation (DIC) or foil crack gauges. A number of teeth were notched to allow fatigue cracks to be initiated promptly.

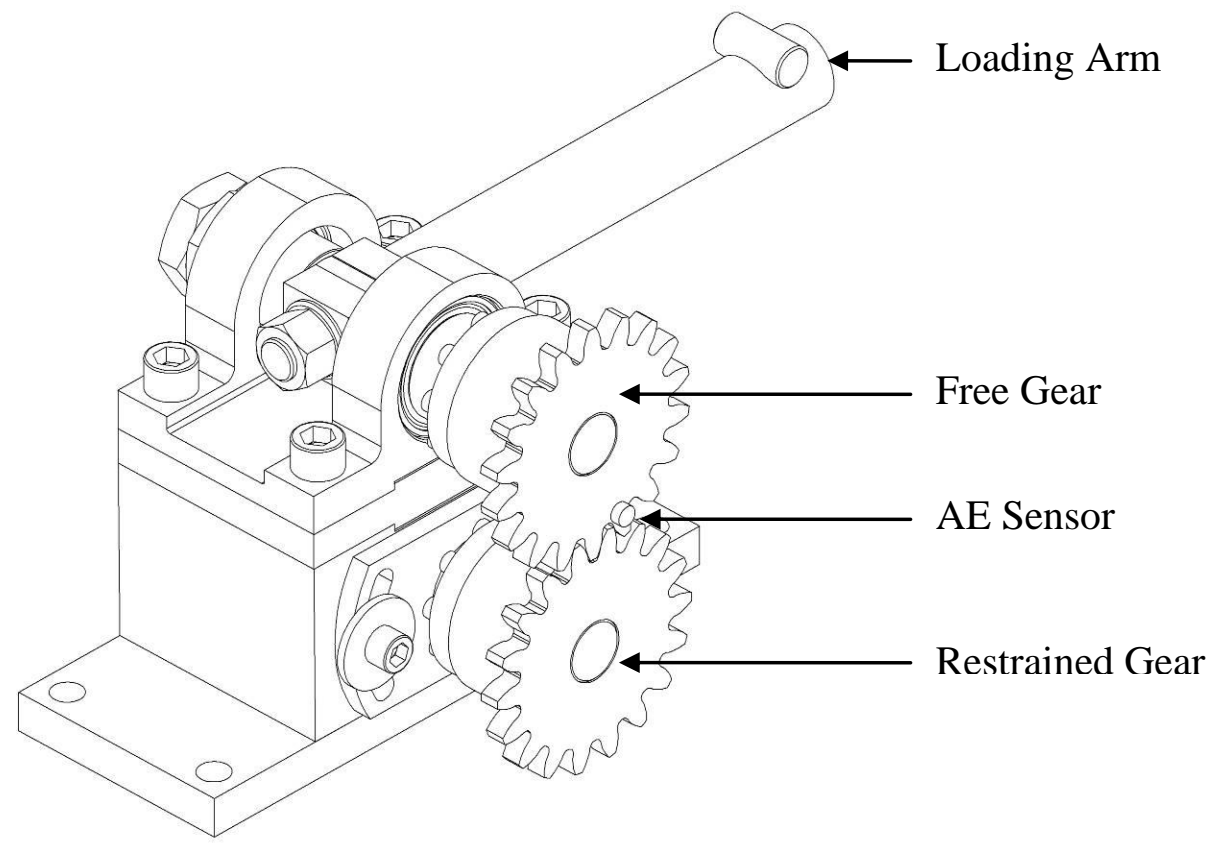

Fig. 1: Test Rig Design

A combination of AE and DIC was used to monitor the fatigue failure of a notched gear tooth. An initial static loading of an undamaged tooth was completed in order to validate an FEA model completed in PATRAN and to assist in determining the appropriate fatigue loading. To validate the $\mathrm{AE}$ results periodically during the investigation the test was stopped, peak load applied and DIC images captured. The gear tooth was loaded from 100 to $1400 \mathrm{~N}$ at $1 \mathrm{~Hz}$. 
During the fatigue investigation, a Physical Acoustics Limited (PAL) Pico (200-750 kHz) AE sensor was coupled and attached to the adjacent gear tooth using cyanoacrylate. Discrete AE data using a threshold of $45 \mathrm{~dB}$ was captured using a PAL PCI 2 system at a sample rate of $2 \mathrm{MHz}$ for the duration of the investigation. Furthermore, after each stop in testing to capture DIC images, a wavestream for 2 seconds (to ensure a complete load cycle was captured) at $2 \mathrm{MHz}$ was captured. Wavestreaming continuously records the output of the sensor and is independent of threshold.

\section{Results and Discussion}

A comparison of the DIC results at $1400 \mathrm{~N}$ (static load) with the FEA model results (at the same load) for strain in the Y-direction is shown in Fig. 2. When comparing the DIC with the FEA results, it must be noted that the DIC cannot analyse the entire tooth due to the method of averaging over a subset of pixels used by the image correlation software to improve accuracy. However, if strain is compared at corresponding positions, there is a broad agreement. On the edge of the tooth root fillets the DIC reports +/- 1200 microstrain whilst at the corresponding positions on the FEA, the strain is between 1100 and 1300 microstrain. It should also be noted that the DIC does not capture the strains due to the Hertzian contact which results in a significantly smaller maximum tensile and compressive strains to be reported. This will be explored in future investigations, and it is unclear currently whether this is due to the resolution of the DIC or some misalignment in the test rig.
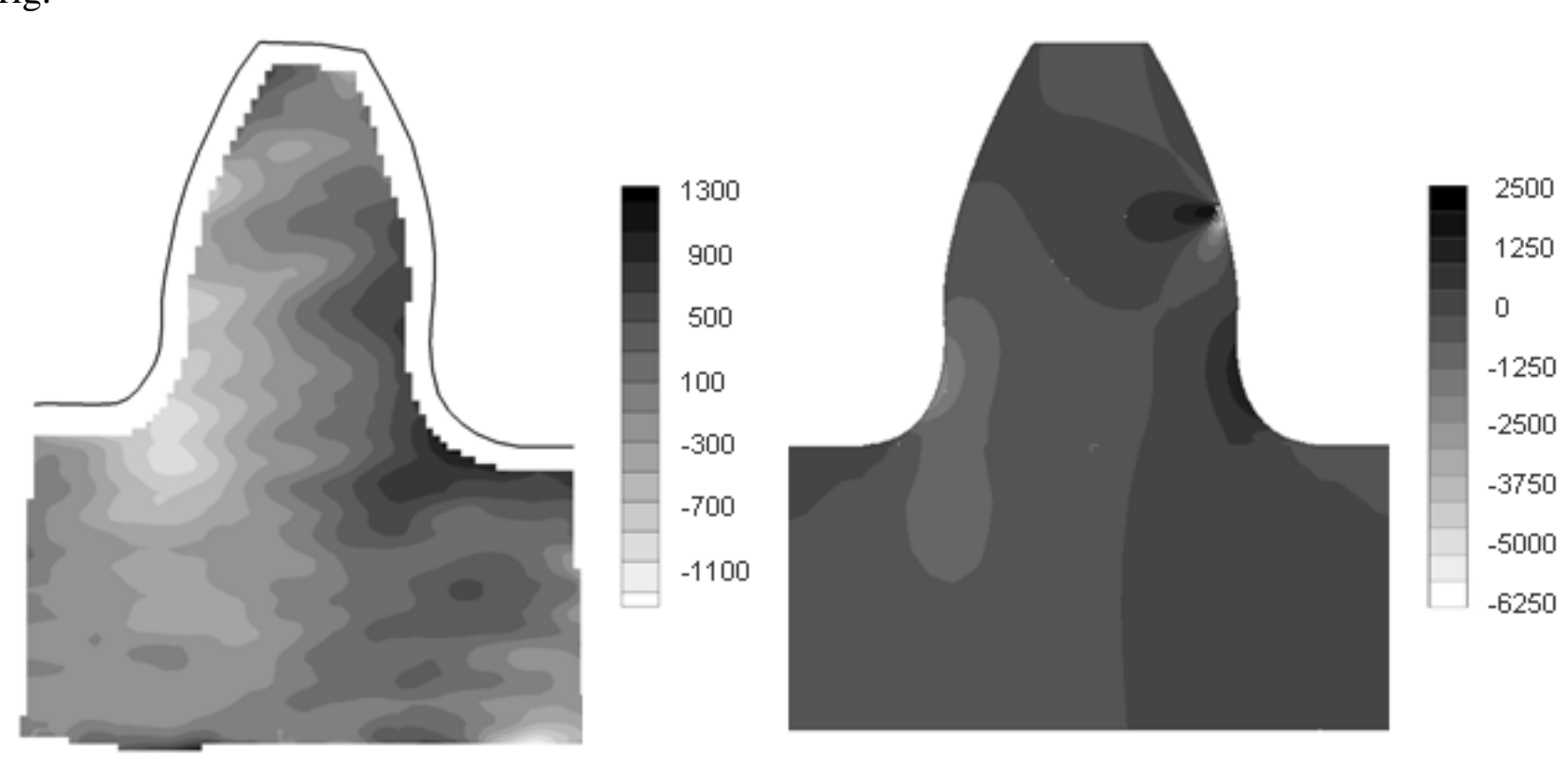

Fig 2. Comparison of y-strain from DIC (left) and FEA (right)

A fatigue crack of approximately $2 \mathrm{~mm}$ had propagated through the gear tooth after $23 \mathrm{k}$ cycles and the test was stopped. The DIC data was analysed after each block of fatigue testing whilst the AE data analysis was completed post test. Fig. 3 shows the DIC images captured periodically through the investigation. A key to the strain contours is provided but should not be considered to be a true representation of the actual magnitude of the strain values around the crack position due to the calculation method employed by the image correlation software. The measurements are calculated by examining the relative movements of adjacent pixels in the images; however whilst the DIC technique captures accurately the relatively large pixel displacements around the crack mouth, the resulting calculated strain is an over-estimation as these displacements are largely caused by crack opening as opposed to elastic strain.

The strain contours clearly show the growth of a high stress region propagating from the notch in the tooth as the test progresses due to increasing crack length and furthermore a subtle difference 
can be noted after $1 \mathrm{k}$ cycles suggesting the crack had already started propagating at that point. A further method of identifying crack growth based on the DIC measurements was employed. The relative movement of two pixels either side of the notch were used to provide a crack mouth opening displacement (CMOD) measurement in order to validate and support the understanding of the collected AE data.

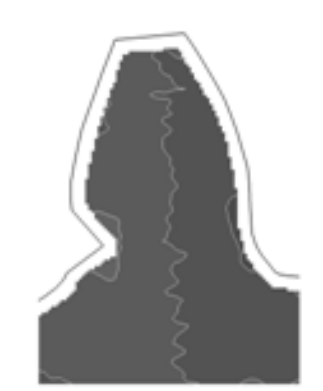

0 Cycles

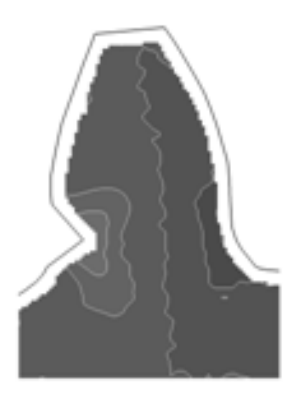

$16 \mathrm{k}$ Cycles

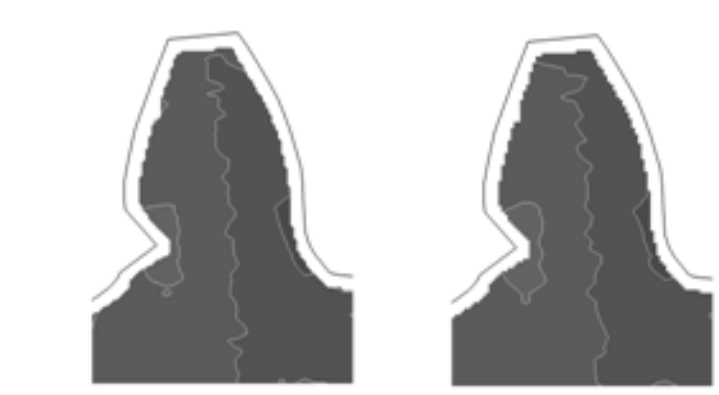

$1 \mathrm{k}$ cycles

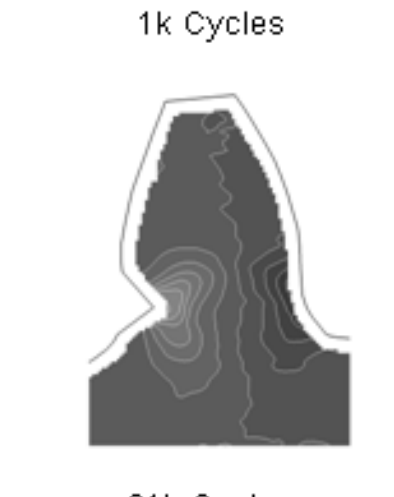

$21 \mathrm{k}$ Cycles

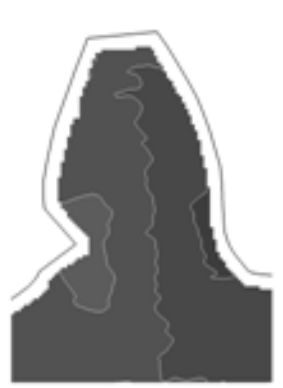

$11 \mathrm{k}$ Cycles

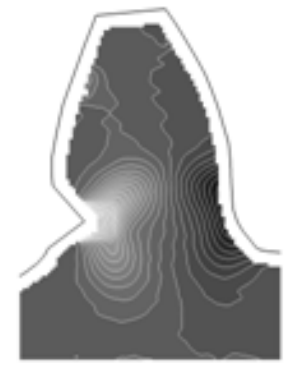

$22 \mathrm{k}$ Cycles

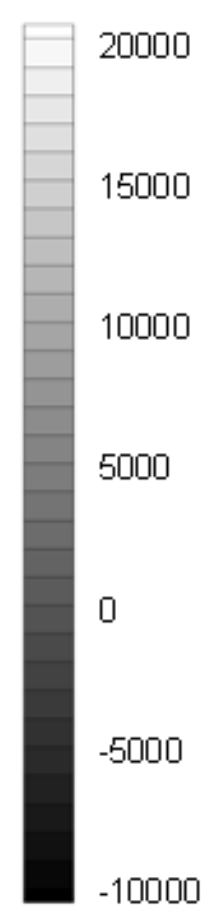

Fig 3. Development of microstrain during fatigue loading

Fig. 4 shows the CMOD measurement determined using the DIC system. Using an optical measurement offers distinct advantages over a foil crack gauge or an actual CMOD measurement. A non-contact measurement technique does not introduce sources of $\mathrm{AE}$ due to friction or glue cracking which would occur in the crack region, thus providing false or misleading results. The cumulative acoustic energy detected by the AE sensor is also shown in Fig. 4. The plot demonstrates that the $\mathrm{AE}$ is detecting the crack growth and that at the point of a large increase in crack length after $21 \mathrm{k}$ cycles, there is a corresponding large increase in detected energy. Furthermore, at the very onset of the test there is little AE detected until approximately 200 cycles suggesting that this is the point of crack initiation.

A simple measurement of energy will be insufficient for detecting fractures in a high noise environment associated with rotating machinery due its lack of sensitivity but it clearly demonstrates that $\mathrm{AE}$ can detect cracking of gear teeth without the presence of rotating noise. Subsequent tests with a sensor mounted on bearing housing have shown the AE method to still be capable of detecting tooth cracking. This work will be the subject of a further publication.

Fig. 5 shows the AE wave-streams captured at discrete points throughout the investigation with the load cycle overlaid. The initial wave-stream prior to cracking in Fig 5a shows only low level background noise, however as soon as cracking has occurred, based on the DIC and traditional AE data, the wave-streams exhibit clear bursts of activity that occur at peak loads - further demonstrating that the $\mathrm{AE}$ is detecting the crack growth in the gear. This again is probably insufficient for detecting fractures in a high noise environment and offers very little scope for providing an automated system. 


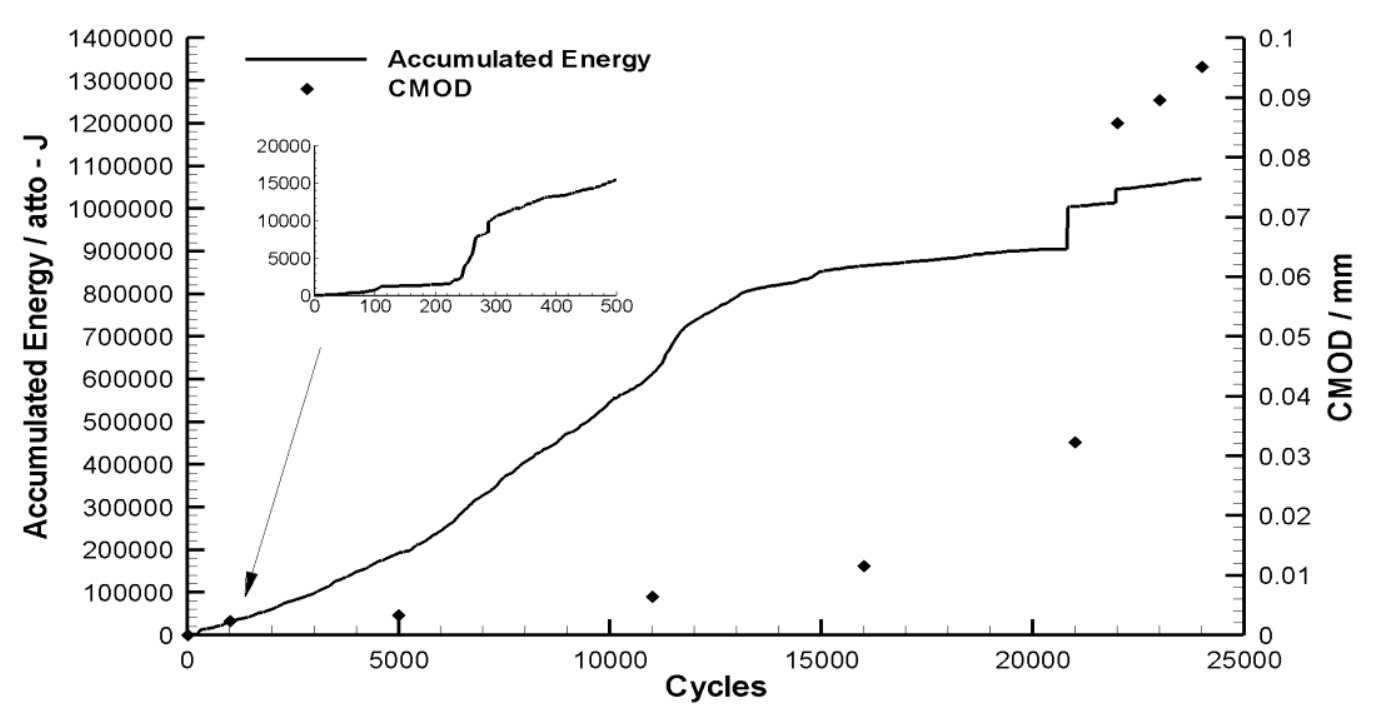

Fig 4 Recorded AE data compared with crack mouth opening measurements
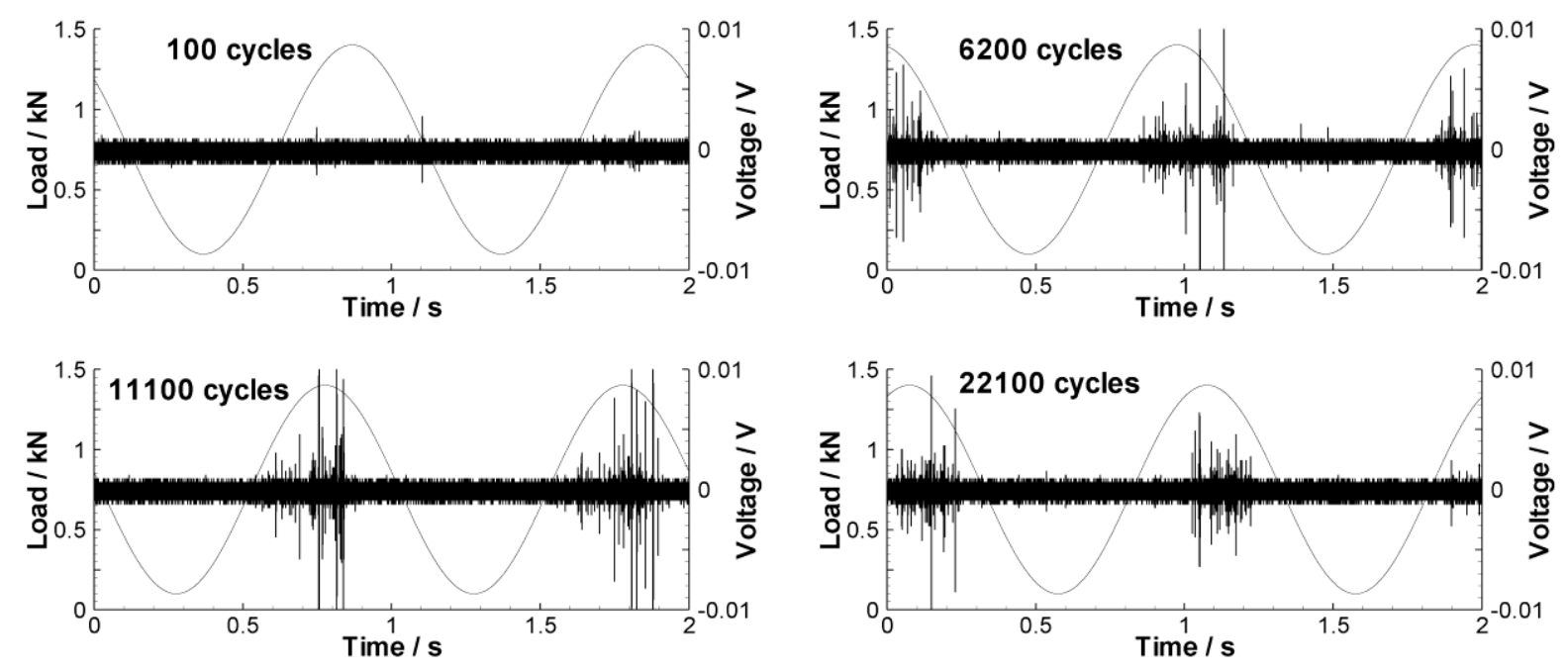

Fig. 5 Wave streaming data captured at discreet points during the investigation

A further method of exploring the data is shown in Fig. 6. A wavelet transform is shown of a 1 second sample which contains the peak load, prior to cracking and at $11.1 \mathrm{k}$ cycles. Wavelet transforms offer significant benefit in analysing the collected AE data. The wavelet transform analyses a signal in the time domain for its frequency content thus providing information of the content and temporal position at frequencies within a signal. This is clearly advantageous as it is easier to identify differing sources within the dataset. Regions of cracking will have different frequencies to that of rotating noise.

This offers a great possibility for developing an automated system for identifying fractures in gear teeth. By using either a novelty or cross correlation technique of a wavelet transform of a wave-stream recorded on one complete rotation, new sources can be identified and flagged as suspect cracks. This could be further enhanced by identifying frequency bands for cracks and use them as a further crack indicator. The techniques discussed will be the focus of future tests and research publications. 

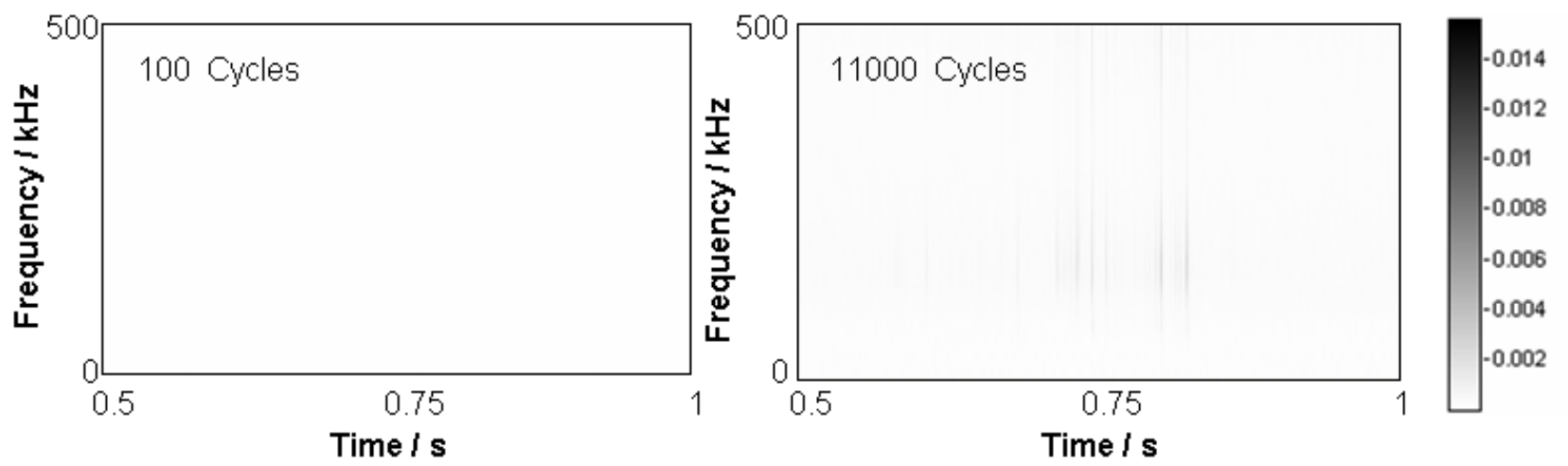

Fig. 6 Wavelet transforms of wave-streams centred around peak load (plots have same peak scale)

\section{Conclusions}

The completed work has demonstrated the capabilities of AE for detecting fatigue fractures in gear teeth. Furthermore techniques for developing an automated system have been explored and demonstrate real potential for designing an automated gear health monitoring system

\section{Acknowledgements}

The authors would like to acknowledge the support and advice provided by the technical staff of Cardiff School of Engineering with particular thanks to Paul Malpas who manufactured the test rig.

\section{References}

[1] Wong AK, Vibration-based helicopter health monitoring, (DSTO, 2001)

[2] Decker HJ, Gear crack detection using tooth analysis, (NASA-TM2002-211491, 2002)

[3] Decker HJ, Crack detection of aerospace quality gears, (NASA-TM2001-211492, 2002)

[4] Larder BD, Helicopter HUM/FDR: Benefits and Developments, (American Helicopter Society, Montreal, Canada, 1999)

[5] McColl J, Overview of transmissions HUM performance in UK, North Sea Helicopter Operations, (Institution of Mechanical Engineers, London n.d.)

[6] Loutas TH et al., Applied Acoustics Vol. 70 (2009), p. 1148

[7] Holford K M et al., Proc IMechE Part G, Journal of Aerospace Engineering Vol 223 (2009), p. 525

[8] Pullin R et al., Journal of Acoustic Emission Vol 25 (2007), p. 215

[9] Hensman J J et al., Applied Mechanics and Materials Vols 13 - 14 (2008), p. 251

[10] Toutountzakis T, Tan CK, Mba D, NDT E Int Vol 38(1) (2005), p.27

[11] Tan CK and Mba D, Tribol. Int. Vol 38(5) (2005), p. 469

[12] Tan CK and Mba D, Proc Inst Mech Eng Part J J. Eng Tribol Vol 219 (2005), p. 401

[13] Loutas TH et al., Trans ASME J Vibration and Acoustics Vol 130 (2008)

[14] Corus Northern Engineering Services, PCM Brochure 001 “Aquilla AE Pro”, (2009) 